\title{
Organismes d'officialisation, dictionnaires et médias : le triangle des Bermudes de la francisation
}

\author{
Vincent, Nadine \\ Université de Sherbrooke \\ nadine.vincent@usherbrooke.ca
}

\begin{abstract}
Dans certains États francophones, des organismes gouvernementaux ont le mandat de proposer ou de recommander des équivalents français aux anglicismes qui prolifèrent dans la langue nationale. Quel est alors le rôle des dictionnaires? Relayer ces recommandations ou attendre que les termes francisés s'implantent dans l'usage? De même, les journalistes doivent-ils utiliser les néologismes recommandés dès leur officialisation, quitte à utiliser des mots inconnus de leur lectorat, ou doivent-ils plutôt attendre que ces emplois soient bien implantés dans l'usage et attestés par les dictionnaires? Dans ce triangle d'acteurs stratégiques, quel est le rôle et quelle est l'influence réelle de chacun dans l'implantation de mots français pour remplacer des anglicismes? C'est ce que nous tenterons d'identifier dans cette étude, en comparant les façons de faire en France et au Québec, pour les anglicismes du domaine des télécommunications e-mail, chat, et podcasting. Pour chacun d'eux, nous analyserons l'action des organismes d'officialisation, la réception des dictionnaires et la diffusion dans l'usage des anglicismes et de leurs équivalents à partir d'un corpus journalistique.
\end{abstract}

\section{Les organismes d'officialisation}

En France, l'Académie française veille depuis 1635 sur le destin de la langue française.

La principale fonction de l'Académie sera de travailler, avec tout le soin et toute la diligence possibles, à donner des règles certaines à notre langue et à la rendre pure, éloquente et capable de traiter les arts et les sciences. » (Article 24 des statuts. $)^{1}$

Quelque trois siècles plus tard, au début des années 1960, naît la notion de francophonie, notamment à la suite de l'indépendance de plusieurs États africains francophones. Le besoin d'une nouvelle instance se fait sentir.

C'est par un décret daté du 31 mars 1966 que le général de Gaulle et Georges Pompidou créent le Haut Comité pour la défense et l'expansion de la langue française, premier élément de la politique de la langue mise en place par les pouvoirs publics. (Chansou, $1997: 25$ ).

À partir de 1970, des commissions de terminologie, mises sur pied dans différents ministères, ont comme mission d'assurer la présence du français dans les domaines de spécialités, et doivent proposer des équivalents aux différents anglicismes qui tendent à s'installer.

Parmi les termes de création ancienne on peut citer logiciel, proposé par la commission de l'informatique dès 1970. Il est formé avec le suffixe -iel de matériel, traduction de l'anglais hardware. Ainsi le français s'est-il doté d'un équivalent au mot software, dont l'espagnol ou l'italien, par exemple, sont aujourd'hui dépourvus. ${ }^{2}$ 
Ce Haut Comité est modifié au cours des différentes législations et donne naissance en 1996, entre autres organismes, à la Commission générale de terminologie et de néologie, qui relève, encore à ce jour, du bureau du Premier ministre, et dont le rôle est de coordonner les travaux des différentes commissions de terminologie.

Les termes recommandés par la Commission générale sont publiés au Journal officiel de la République française; ils ne sont d'usage obligatoire que dans les administrations et les établissements de l'État mais ils peuvent servir de référence, en particulier pour les traducteurs et les rédacteurs techniques. ${ }^{3}$

Conformément au décret ${ }^{\circ} 96-602$ du 3 juillet 1996 relatif à l'enrichissement de la langue française, ces recommandations doivent cependant obtenir l'aval de la vieille dame du quai Conti.

Les termes, expressions et définitions proposés par la commission générale ne peuvent être publiés au Journal officiel sans l'accord de l'Académie française. Si celle-ci n'a pas formulé d'avis dans un délai de quatre mois à compter de sa saisine, son accord est réputé acquis. ${ }^{4}$

Au Québec, l'Office québécois de la langue française (OQLF) $)^{5}$ est créé en 1961. Comme pour le Haut Comité pour la défense et l'expansion de la langue française, son mandat est modifié au fil des législations. L'une de ses missions touche la francisation des termes spécialisés.

La Charte de la langue française adoptée par l'Assemblée nationale du Québec en 1977 et modifiée le 12 juin 2002 a conféré à l'Office québécois de la langue française la mission de définir et de conduire la politique québécoise en matière d'officialisation linguistique, de terminologie ainsi que de francisation de l'Administration et des entreprises ${ }^{6}$.

Pour ce faire, un Comité d'officialisation linguistique procède à la recommandation ou à la normalisation de termes ou expressions.

Recommandation: La recommandation des termes et expressions est conçue comme une mesure incitative puisque les termes et expressions recommandés ne sont pas assujettis à un usage obligatoire par l'Administration.

Normalisation : La normalisation des termes et expressions a un effet contraignant, puisque «dès la publication à la Gazette officielle du Québec des termes et expressions normalisés par l'Office, leur emploi devient obligatoire dans les textes, les documents et l'affichage émanant de l'Administration ainsi que dans les contrats auxquels elle est partie, dans les ouvrages d'enseignement, de formation ou de recherche publiés en français au Québec et approuvés par le ministre de l'Éducation » (art. 118). (Office québécois de la langue française, $2004: 5$ ).

Dans la majorité des cas, les équivalents français aux anglicismes ne sont ni recommandés ni normalisés, ils ne sont que proposés, et c'est l'usage qui décide s'ils s'implanteront ou non. C'est d'ailleurs le cas pour les trois séries à l'étude dans cet article (voir tableau 1). Alors que la France a officialisé des termes pour remplacer les emprunts à l'anglais, le Québec s'est contenté d'en proposer. Pour la première des trois séries (e-mail/mail et courriel), la forme francisée est la même des deux côtés de l'Atlantique, alors que pour les deux autres (chat/tchat, dialogue en ligne et clavardage; podcasting, diffusion pour baladeur et baladodiffusion) les formes francisées diffèrent en France et au Québec. 


\begin{tabular}{|c|c|c|}
\hline & $\begin{array}{c}\text { France } \\
\text { (officialisation) }\end{array}$ & $\begin{array}{c}\text { Québec } \\
\text { (création/proposition) }\end{array}$ \\
\hline e-mail/mail & $\begin{array}{c}\text { courriel } \\
20 \text { juin } 2003\end{array}$ & $\begin{array}{c}\text { courriel } \\
1990\end{array}$ \\
\hline chat/tchat & $\begin{array}{c}\text { dialogue en ligne } \\
5 \text { avril 2006 }\end{array}$ & $\begin{array}{c}\text { clavardage } \\
\text { octobre 1997 }\end{array}$ \\
\hline podcasting & $\begin{array}{c}\text { diffusion pour baladeur } \\
\text { 25 mars 2006 }\end{array}$ & $\begin{array}{c}\text { baladodiffusion } \\
\text { octobre 2004 }\end{array}$ \\
\hline
\end{tabular}

Tableau 1 : francisation des anglicismes e-mail, chat et podcasting en France et au Québec

\section{Traitement des anglicismes et des formes francisées dans les dictionnaires}

\subsection{En France}

Pour rendre compte du traitement des anglicismes dans les dictionnaires français, nous avons consulté $L e$ Petit Robert 2014 et Le Grand Robert 2013, d'abord parce que ces deux ouvrages énoncent clairement leur position sur les emprunts à l'anglais, l'usage et les officialisations; ensuite parce qu'ils proposent des descriptions distinctes, malgré leur évidente proximité.

Selon le tableau des abréviations des pages liminaires du Petit Robert, la marque «ANGLIC. » qui accompagne certains mots ou certaines acceptions identifie un anglicisme, c'est-à-dire un " mot anglais [...] employé en français et critiqué comme emprunt abusif ou inutile ${ }^{7} »$. C'est ensuite au lecteur de décider s'il emploiera ou non les mots ainsi marqués. Chose certaine, les rédacteurs du dictionnaire ne s'en privent pas, eux, puisqu'on retrouve ces anglicismes utilisés pour définir d'autres mots. Ainsi, le terme de golf par, identifié comme anglicisme et définit comme le «nombre de coups nécessaires pour réussir un trou ${ }^{8}$ » est utilisé dans la définition d'albatros : «Au golf, Trou réalisé en trois coups de moins que le par $^{9} »$. On peut donc difficilement considérer que la marque ANGLIC. condamne l'emploi d'un mot. Certains emplois d'origine anglaise sont accompagnés du double marquage " ANGLIC. CRITIQUÉ », par exemple positionnement, et ne sont pas davantage proscrits du dictionnaire puisqu'ils sont aussi utilisés dans la définition d'autres mots, par exemple tabulation: «Espacement prédéfini sur la ligne de frappe; positionnement du chariot, du curseur qui lui correspond. ». Enfin, certains emplois sont accompagnés de l'indicateur «FAUX ANGLIC. ${ }^{10}$ » (non défini dans le tableau des abréviations), qui tient plutôt de l'étymologie que du marquage normatif.

Qu'il soit critiqué ou non, qu'il fasse double emploi ou non avec un mot français existant, c'est d'abord la vitalité d'un anglicisme dans l'usage, authentifié par sa présence dans les médias, qui est la voie la plus sûre pour son entrée dans Le Petit Robert. «Le mot doit circuler de manière observable et indiscutable dans la société. Son écho dans la presse, dans les conversations, à la radio, à la télévision...sont des gages de recevabilité. $"{ }^{11}$ Dans le préface du Nouveau Petit Robert (1993), Josette Rey-Debove et Alain Rey expliquent clairement l'influence respective des organismes d'officialisation et de l'usage.

Dans le domaine des terminologies, des commissions ministérielles se réunissent en France, des institutions ont été créées au Québec pour proposer des mots français en remplacement des anglicismes, et parfois l'entreprise est couronnée de succès complet (ordinateur pour computer) ou relatif (courriel pour e-mail). Nous avons signalé comme tels les anglicismes et américanismes récents et indiqué le mot français correspondant proposé par les commissions de terminologie (recommandation officielle), sans faire apparaître à la nomenclature ce qui n'est pas attesté par l'usage. Comme on 
l'a déjà dit, la vocation du Petit Robert n'est pas de légiférer, mais d'observer la langue en attirant l'attention sur les problèmes. ${ }^{12}$

Vingt ans après sa parution, et dix ans après que la Commission générale de terminologie et de néologie ait recommandé l'emploi de courriel pour remplacer e-mail ${ }^{13}$, cette préface est toujours reproduite dans les derniers millésimes du Petit Robert. Cependant, à l'intérieur des pages du dictionnaire, les données sont mises à jour. L'indication de la recommandation officielle de courriel est présente dans le dictionnaire depuis l'édition de $2007^{14}$, mais uniquement dans l'article e-mail, entre parenthèses à la suite du renvoi à courriel (voir tableau 2). L'information n'est présente ni dans l'article courriel ni dans l'article mail, tous deux renvoyant à $e$-mail.

Dans Le Grand Robert 2013, l'information de la recommandation officielle de courriel est absente des articles e-mail, mail et courriel. En fait, ces trois articles n'ont pas été modifiés depuis l'édition de 2001, et on indique encore, sous courriel, que ce mot est « en usage en français du Canada », et sous e-mail, que « Courriel [...] est utilisé au Québec et en Belgique ». Comprenne qui pourra.

La préface de la dernière édition du Grand Robert ne nous donne aucune information sur le traitement des anglicismes, mais on trouve les indications recherchées dans la préface de la deuxième édition (19842001), signée Alain Rey, et reproduite dans l'édition numérique de 2013.

Pour une catégorie d'emprunts particulièrement - excessivement abondante, les anglicismes, on a suivi [...] une politique d'objectivité, en distinguant simplement les emprunts bien intégrés [...] qui sont traités sans autre commentaire, des innombrables emprunts récents ou mal intégrés, et qui sont alors qualifiés d'«anglicismes» (abrégé en anglic.). Cette « marque » signifie que ces mots ne sont pas unanimement acceptés et font parfois l'objet d'une décision officielle de francisation — qui est signalée. ${ }^{15}$

Cette décision est parfois signalée, il est vrai, mais elle n'est visiblement pas considérée comme une donnée prioritaire à intégrer aux mises à jour, ainsi que nous l'avons vu avec courriel. De même, Le Grand Robert 2013 indique encore que causette est l' «équivalent proposé » pour remplacer l'anglicisme chat (parfois écrit tchat), bien que cette recommandation ait été remplacée par dialogue en ligne en avril $2006^{16}$ (tableau 2). On précisait alors au Journal officiel « attention : Cette publication annule et remplace celle du terme "causette" au Journal officiel du 16 mars $1999 »^{17}$. Par ailleurs, toute allusion au sens informatique de causette est absente de l'article causette lui-même dans Le Grand Robert 2013, ainsi que dans l'édition de 2001. Comme pour e-mail et courriel, aucune mise à jour n'a donc été faite pour ces mots entre les deux éditions. De son côté, Le Petit Robert a remplacé causette par dialogue en ligne comme recommandation officielle de chat dès 2008 et a éliminé de son article causette le sens informatique qui y apparaissait précédemment. 


\begin{tabular}{|c|c|c|c|c|c|c|}
\hline & \multicolumn{3}{|c|}{ Le Petit Robert 2014} & \multicolumn{3}{|c|}{ Le Grand Robert 2013} \\
\hline & marque(s) & renvoi(s) & officialisation & marque(s) & renvoi(s) & officialisation \\
\hline e-mail & ANGLIC. & $\begin{array}{c}\text { courriel } \\
\text { (recommandation } \\
\text { officielle) }\end{array}$ & - & Anglic. & - & - \\
\hline mail & ANGLIC. & $\begin{array}{c}\text { courriel } \\
\text { e-mail }\end{array}$ & - & Anglic. & $\begin{array}{c}\text { e-mail } \\
\text { courriel }\end{array}$ & - \\
\hline courriel & - & e-mail & - & $\begin{array}{c}\text { (En usage en } \\
\text { franç. du Canada) }\end{array}$ & - & - \\
\hline chat & $\begin{array}{l}\text { ANGLIC. } \\
\text { INFORM. }\end{array}$ & $\begin{array}{l}\text { RÉGIONAL } \\
\text { clavardage }\end{array}$ & $\begin{array}{l}\text { recommandation } \\
\text { officielle : } \\
\text { dialogue en ligne. }\end{array}$ & Anglic. & - & $\begin{array}{c}\text { Équivalent } \\
\text { proposé : causette }\end{array}$ \\
\hline $\begin{array}{c}\text { causette } \\
\text { (sens informatique) }\end{array}$ & - & - & - & - & - & - \\
\hline dialogue en ligne & - & - & $\begin{array}{c}\text { recommandation } \\
\text { officielle pour } \\
\text { 1'anglic. chat }\end{array}$ & - & - & - \\
\hline clavardage & $\begin{array}{l}\text { RÉGIONAL } \\
\text { (Canada) }\end{array}$ & chat & & Franç. du Canada & $\begin{array}{c}\text { chat } \\
\text { (anglic.) }\end{array}$ & \\
\hline podcast & ANGLIC. & baladodiffusion & $\begin{array}{l}\text { recommandation } \\
\text { officielle : } \\
\text { diffusion pour } \\
\text { baladeur } \\
\end{array}$ & Anglic. & - & $\begin{array}{l}\text { Recomm. off. : } \\
\text { diffusion pour } \\
\text { baladeur }\end{array}$ \\
\hline podcasting & - & - & - & - & - & - \\
\hline $\begin{array}{l}\text { diffusion pour } \\
\text { baladeur }\end{array}$ & - & - & $\begin{array}{l}\text { recommandation } \\
\text { officielle pour } \\
\text { podcasting }\end{array}$ & - & - & - \\
\hline baladodiffusion & $\begin{array}{c}\text { RARE } \\
\text { (courant au } \\
\text { Canada) }\end{array}$ & podcast & - & $\begin{array}{c}\text { Rare } \\
\text { (cour. au Canada) }\end{array}$ & $\begin{array}{l}\text { anglic. } \\
\text { podcast }\end{array}$ & - \\
\hline
\end{tabular}

Tableau 2 : Traitement des anglicismes e-mail, chat, podcast et podcasting et de leurs équivalents dans Le Petit Robert 2014 et Le Grand Robert 2013.

Pour la troisième série, les deux dictionnaires indiquent la recommandation officielle diffusion pour baladeur, sous l'anglicisme podcast. C'était cependant pour podcasting que la recommandation avait été faite, ainsi que l'indique le Journal officiel du 25 mars 2006, de même que Le Petit Robert sous baladeur: «diffusion pour baladeur: recommandation officielle pour podcasting. » Podcasting est absent de la nomenclature des deux dictionnaires et Le Grand Robert n'atteste le terme francisé ni sous diffusion ni sous baladeur.

En fait, les deux dictionnaires indiquent deux acceptions pour podcast : le « fichier diffusé par Internet », et le «mode de diffusion de ce fichier ». La Commission générale de terminologie et de néologie n'a officialisé qu'un terme français, diffusion pour baladeur, qui correspond à la seconde acception et au mot anglais podcasting. Le Québec, par le biais de l'OQLF, a proposé baladodiffusion pour le mode de diffusion, et balado, pour le fichier diffusé. Les dictionnaires Robert n'attestent que baladodiffusion avec l'unique sens de «mode de diffusion de ce fichier » et taisent l'existence de balado.

Pour terminer, Le Petit Robert indique généralement les recommandations officielles à deux endroits, sous le mot anglais et sous la forme officialisée. Celle-ci n'est pas définie si elle ne s'est pas implantée dans l'usage, comme ce semble être le cas pour dialogue en ligne et diffusion pour baladeur. Le mot courriel constitue un cas particulier puisqu'il est entré dans Le Petit Robert par une porte dérobée, apparaissant d'abord en entrée-cachée comme un emploi non hexagonal sous e-mail : «REM. Courriel (contract. de courrier électronique) est un équivalent possible, utilisé au Québec et en Belgique. ${ }^{18}{ }$. Puis, 
il fait son apparition à la nomenclature, mais sans marque géographique. ${ }^{19}$ Depuis le millésime 2006, on peut apprendre à la suite de son renvoi sous e-mail qu'il a fait l'objet d'une recommandation officielle. Quant au Grand Robert, il ne nous est pas possible d'identifier une méthodologie le moindrement systématique pour l'indication des officialisations.

Notons enfin que trois équivalents français utilisés au Québec sont à la nomenclature des deux dictionnaires de la maison Robert, et indiqués en renvoi dans Le Petit Robert, uniquement sous le mot anglais : courriel sous e-mail, clavardage sous chat et baladodiffusion sous la seconde acception de podcast. Le Grand Robert se contente de renvoyer à courriel sous mail, variante de e-mail. Dans ce dictionnaire, il apparaît donc impossible de repérer les emplois québécois si on ne les connaît pas déjà. Le Journal officiel avait indiqué l'usage québécois pour un seul de ces trois mots : « Au Québec, on emploie le terme "baladodiffusion"20 ». Le Grand Robert relaie l'information en remarque dans son article baladodiffusion : «Le mot est préconisé au Québec par l'Office de la langue française ${ }^{21}$. »

\subsection{Au Québec}

Sans entrer dans une histoire de la lexicographie québécoise, disons simplement que la façon de traiter les anglicismes peut être au Québec un des éléments déterminants dans la survie ou non d'un dictionnaire. À titre d'exemple, le Dictionnaire québécois d'aujourd'hui, publié en 1992 par la maison Robert, s'est fait reprocher son traitement trop peu normatif.

[Des] journalistes, linguistes ou enseignants ont fait ressortir des faiblesses certaines du côté des marques d'usage, notamment anglicisme. Il se dégage de leurs commentaires que ces marques ne rendaient pas compte assez fidèlement du sentiment linguistique des locuteurs québécois. [...] Les anglicismes n'ont pas toujours été suffisamment identifiés et balisés. [...] Et même si, à la suite de ces critiques, le Robert a décidé d'apporter des rectificatifs dans une seconde édition publiée en 1993, le mal (suscité par les critiques virulentes) était fait. (Poisson, $2008: 285$ )

Cette relation particulière aux emprunts à l'anglais s'explique bien sûr par la situation géodémographique unique du Québec, îlot de quelque sept millions de francophones au milieu d'un océan de plus 300 millions d'anglophones (au Canada anglais et aux États-Unis), mais aussi par une situation historique où l'anglais a longtemps représenté la langue du conquérant.

Même si les conditions socio-économiques des Québécois et leur niveau d'instruction se sont considérables transformés [...], ils restent profondément marqués par cette histoire sociale qui a associé l'anglicisme à l'ignorance, la pauvreté et la domination subie. (Bouchard, 1999 : 15)

Cette sensibilité québécoise explique que les récents dictionnaires faits au Québec jugent plus prudents de tenir compte de la position de l'Office québécois de la langue française sur les anglicismes, comme en témoigne le dernier né d'entre eux, le dictionnaire Usito 22 (tableau 3). 


\begin{tabular}{|c|c|c|c|}
\hline & \multicolumn{3}{|c|}{ Usito 2013} \\
\hline & marque(s) & renvoi(s) & officialisation \\
\hline e-mail & Anglicisme critiqué & courriel & $\begin{array}{l}\text { L'emploi de courriel a fait l'objet d'une } \\
\text { recommandation officielle en France }\end{array}$ \\
\hline mail & Anglicisme critiqué & courriel & $\begin{array}{l}\text { L'emploi de courriel a fait l'objet d'une } \\
\text { recommandation officielle en France }\end{array}$ \\
\hline courriel & - & - & $\begin{array}{l}\text { Réputé d'origine québécoise, l'emploi de courriel a } \\
\text { fait l'objet d'une recommandation officielle en France. }\end{array}$ \\
\hline chat & Anglicisme critiqué & $\begin{array}{c}\text { clavardage } \\
\text { dialogue en ligne }\end{array}$ & $\begin{array}{l}\text { L'emploi de dialogue en ligne a fait l'objet d'une } \\
\text { recommandation officielle en France. }\end{array}$ \\
\hline $\begin{array}{c}\text { causette } \\
\text { (sens informatique) }\end{array}$ & - & 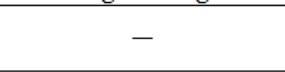 & ( \\
\hline dialogue en ligne & UF & clavardage & $\begin{array}{l}\text { L'emploi de dialogue en ligne a fait l'objet d'une } \\
\text { recommandation officielle en France }\end{array}$ \\
\hline clavardage & UQ & dialogue en ligne & $\begin{array}{l}\text { L'emploi de dialogue en ligne a fait l'objet d'une } \\
\text { recommandation officielle en France. }\end{array}$ \\
\hline podcast & Anglicisme critiqué & balado & - \\
\hline podcasting & Anglicisme critiqué & $\begin{array}{l}\text { baladodiffusion } \\
\text { diffusion pour baladeur }\end{array}$ & $\begin{array}{l}\text { L'emploi de diffusion pour baladeur a fait l'objet } \\
\text { d'une recommandation officielle en France. }\end{array}$ \\
\hline $\begin{array}{l}\text { diffusion pour } \\
\text { baladeur }\end{array}$ & UF & baladodiffusion & $\begin{array}{l}\text { L'emploi de diffusion pour baladeur a fait l'objet } \\
\text { d'une recommandation officielle en France. }\end{array}$ \\
\hline balado & UQ & - & - \\
\hline baladodiffusion & UQ & diffusion pour baladeur & $\begin{array}{l}\text { L'emploi de diffusion pour baladeur a fait l'objet } \\
\text { d'une recommandation officielle en France. }\end{array}$ \\
\hline
\end{tabular}

Tableau 3 : Traitement des anglicismes e-mail, chat, podcast et podcasting et de leurs équivalents dans Usito.

Ce dictionnaire indique systématiquement les officialisations faites en France sous le mot anglais, sous le terme officialisé et sous le mot québécois. Comme dans les trois séries qui nous intéressent, l'Office québécois de la langue française n'a fait ni recommandation ni normalisation, aucune officialisation québécoise n'est indiquée, mais les mots spécifiques à la France ou au Québec sont identifiés au moyen des marques UF (emploi caractéristique de l'usage en France) et UQ (emploi caractéristique de l'usage au Québec). De plus, les termes francisés sont en renvois sous chacun des mots de la série, alors que les anglicismes critiqués apparaissent à la nomenclature mais sont absents des renvois.

\section{Anglicismes et formes francisées dans la presse écrite}

Pour évaluer la vitalité des anglicismes et la pénétration des officialisations dans les médias écrits, nous avons consulté la banque de données Eureka.cc ${ }^{23}$, qui regroupe des milliers de sources journalistiques (articles de journaux, dépêches d'agences de presse, blogues, etc.). Cette consultation a été faite à titre indicatif seulement, les résultats devant être interprétés comme un simple aperçu de tendances. Il faudrait une analyse beaucoup plus fine pour identifier l'usage exact des différents emplois. À titre d'exemple, des documents sont régulièrement dédoublés dans la banque de données, et un même article, publié dans la version papier et sur le site Internet d'une publication, apparaîtra deux fois. De même, une dépêche reprise par différents journaux sera comptabilisée à chacune de ses parutions. Un filtrage des documents serait donc utile pour éliminer les doublons. Par ailleurs, il pourrait être intéressant de distinguer les emplois intégrés des emplois qui font l'objet de commentaires dans l'article. Ainsi, la seule occurrence de diffusion pour baladeur qui apparait dans le sous-corpus français (voir tableau 4) figure dans un article qui annonce que ce terme vient d'être officialiser pour remplacer podcasting. Il serait donc à classer dans les sujets commentés plutôt que dans la langue utilisée pour commenter ${ }^{24}$. 
Nous avons interrogé la banque Eureka.cc pour avoir une idée de la fréquence de chacun des mots anglais et des équivalents français, dans la presse écrite de France et du Québec, pour les deux dernières années. Le jour de l'interrogation, le sous-corpus français comptait 17196316 documents, alors que le souscorpus québécois en comptait 2435 020. Il ne s'agit donc pas de comparer ces deux sous-corpus, mais bien d'observer pour chacun d'eux comme se répartissent les synonymes disponibles pour nos trois séries d'anglicismes et leurs équivalents.

\begin{tabular}{|c|c|c|}
\hline & $\begin{array}{c}\text { en France } \\
\text { Eureka.cc - Europe (FR) } \\
\text { Corpus total : 17 196 316 }\end{array}$ & $\begin{array}{c}\text { au Québec } \\
\text { Eureka.cc - Canada (FR) } \\
\text { Corpus total : 2 435 020 }\end{array}$ \\
\hline chat & \multicolumn{2}{|c|}{ Trop de parasites } \\
\hline tchat & $\mathbf{1 7 6 2}$ & 16 \\
\hline clavardage & 35 & $\mathbf{1 1 8 9}$ \\
\hline dialogue en ligne & 92 & 11 \\
\hline e-mail & $\mathbf{1 1 6 0 1 4}$ & 1396 \\
\hline mail & $\mathbf{1 3 2} \mathbf{6 8 1}$ & 688 \\
\hline courriel & 140439 & $\mathbf{6 0 9 5 3}$ \\
\hline podcast & 1884 & 8 \\
\hline podcasting & 31 & 98 \\
\hline balado & 5 & 175 \\
\hline baladodiffusion & 116 & 0 \\
\hline diffusion pour baladeur & 1 & \\
\hline
\end{tabular}

Tableau 4. Occurrences d'anglicismes et de leurs équivalents français dans Eureka.cc (12 décembre 2013)

Les résultats obtenus sont intéressants (tableau 4). Malgré la disproportion évidente entre les deux souscorpus, on constate que du côté français, c'est toujours le mot anglais qui est le plus fréquent. Dans le cas de chat/tchat, nous n'avons pu obtenir de résultats satisfaisants pour la graphie chat (en raison de son homographie avec le félin domestique), mais la graphie tchat, fort probablement moins fréquente, sort quand même victorieuse devant ses synonymes francisés. Dans le sous-corpus québécois, c'est au contraire la variante francisée clavardage qui écrase ses concurrentes. Pour la série e-mail, mail et courriel, il faut jumeler les deux formes anglaises dans le sous-corpus français pour arriver à dépasser courriel, solidement implanté. Au Québec, courriel n'a visiblement pas de réels concurrents à l'écrit. Enfin, alors qu'en France le mot anglais podcast semble dominer les usages, la course se fait au Québec entre ce mot anglais et le terme francisé proposé par l'OQLF pour remplacer podcasting: baladodiffusion.

\section{Conclusion : le rôle ambigu du dictionnaire}

C'est à son dictionnaire que l'usager moyen se réfèrera pour trouver une réponse en matière de francisation. Bien rares sont ceux qui consultent les recommandations publiées au Journal officiel en France ou diffusées par l'OQLF au Québec. Compte tenu de ce que nous venons d'observer, est-ce que les dictionnaires fournissent à leurs utilisateurs toutes les données pertinentes? 
le Dictionnaire de l'Académie Française est un dictionnaire d'usage, du bel usage même, et sa position institutionnelle lui fournit une légitimité véritable pour l'introduction des remarques normatives, une légitimité que ne possèdent pas les dictionnaires non institutionnels que sont les Larousse et Robert. Ces derniers, soit ne s'octroient pas le droit d'introduire ce type de remarques, soit, lorsqu'ils le font, adoptent une certaine « neutralité » dans la formulation de ces remarques. (Ch. Rey, p. 82)

Cette «neutralité » génère ce que des utilisateurs non informés pourraient percevoir comme de l'incohérence. La troisième série à l'étude ici est particulièrement éloquente à ce sujet. Ainsi, la Commission générale de terminologie et de néologie a officialisé un terme pour remplacer l'anglicisme podcasting. Le Petit Robert a relayé l'information sous la forme francisée diffusion pour baladeur, mais la consultation que nous avons faite des journaux permet de constater qu'il s'est fié à l'usage pour décider quel anglicisme méritait d'apparaître à la nomenclature, et podcasting n'en faisait pas partie. L'anglicisme podcast, lui, semblait suffisamment fréquent, et utilisé aussi bien dans le sens de « fichier diffusé par Internet », que dans celui de «mode de diffusion de ce fichier ». La seule incohérence que l'on puisse reprocher au Petit Robert est d'avoir indiqué sous la seconde acception de podcast une recommandation officielle pour diffusion pour baladeur, alors que ce n'est pas pour cet anglicisme que la recommandation a été faite. Dans les faits, podcast devient pour cette seconde acception, un synonyme de podcasting, mais mieux implanté. Une remarque aurait pu ici lever l'ambiguïté. Malgré cette rigueur, que comprendra l'usager? Dans une moindre mesure, la même confusion s'observe au Québec, alors que l'OQLF fait une distinction nette et étanche entre balado et baladodiffusion, distinction qui ne s'observe pas aussi clairement dans l'usage pour baladodiffusion. Le dictionnaire Usito atteste donc deux acceptions pour ce dernier mot. Le Grand Robert 2013 est malheureusement hors-course pour les cas étudiés, les informations diffusées étant trop décalées par rapport aux données disponibles en 2013.

Fidèle à ce qu'il indique dans sa préface, Le Petit Robert semble se fier d'abord à l'usage pour décider ce qu'il faudra traiter ou non. Ainsi, les mots e-mail (et sa variante mail), chat et podcast, fréquents en contextes, sont définis, exemplifiés, et accompagnés de renvois. Ils servent de porte d'entrée dans le dictionnaire pour leur série synonymique et les emplois francisés reçoivent un traitement minimal tant qu'ils ne sont pas couramment employés. Or, la presse française semble préférer les anglicismes à leurs équivalents officialisés. Au Québec, probablement en raison du sentiment linguistique des Québécois qui sont réfractaires à la présence d'anglicismes dans la langue standard, on observe une plus grande systématisation dans l'utilisation des formes francisées dans la presse, et un traitement plus critique à l'écart des anglicismes dans les outils de référence, comme le dictionnaire Usito.

Dans ce trio d'acteurs que sont les organismes d'officialisation, les médias et les dictionnaires, on peut donc constater qu'en France chacun joue son rôle selon ses propres objectifs, alors qu'au Québec, tous semblent guidés par la même règle implicite. Dans un cas comme dans l'autre, la question se pose : est-ce le rôle des dictionnaires, publics comme privés, d'expliquer la norme, voire de l'orienter, ou doivent-ils n'en être que les témoins?

\section{BIBLIOGRAPHIE}

Bouchard, Ch. (1999) On n'emprunte qu'aux riches. La valeur sociolinguistique et symbolique des emprunts, Fides. Chansou, M. (1997) «Les politiques de la langue et la législation linguistique en France (1966-1994)», Mots, no 52, p. 23-35. 
Engstrom, Line (2005) Les anglicismes dans le français hexagonal contemporain : analyse d'un corpus de presse, Mémoire de master, Université d'Oslo.

Le Grand Robert de la langue française 2013 (2013), édition numérique, $3^{\mathrm{e}}$ édition, Paris : Dictionnaires Le Robert.

Le Grand Robert de la langue française, dictionnaire alphabétique et analogique de la langue française (2001), $2^{\mathrm{e}}$ édition, Paris : Dictionnaires Le Robert.

Office québécois de la langue française (2007) Politique de l'emprunt linguistique, Québec: Gouvernement du Québec.

Office québécois de la langue française (2004) Politique de l'officialisation linguistique, Québec : Gouvernement du Québec.

Le Petit Robert de la langue française 2014 (2013), édition numérique, Paris : Dictionnaires Le Robert.

Poisson, Esther (2008) «Le Dictionnaire québécois d'aujourd'hui: un jalon important de l'évolution de la lexicographie au Québec», Les dictionnaires de la langue française au Québec, de la Nouvelle-France à aujourd'hui, sous la direction de M.C. Cormier et J.-Cl. Boulanger, Les Presses de l'Université de Montréal, p. $267-$ 290.

Rey, Alain (2007) « Préface », Le Petit Robert de la langue française 2008, Dictionnaires Le Robert.

Rey, Christophe (2012) "Les «Recommandations normatives» de la neuvième édition du Dictionnaire de l'Académie Française », Dictionnaires, norme(s) et sociolinguistique, Paris : L'Harmattan, p. 59-83.

Zanola, Maria Teresa (2008) «Les anglicismes et le français du XXI ${ }^{\mathrm{e}}$ siècle : La fin du franglais? » Synergies Italie, $\mathrm{n}^{\mathrm{o}} 4$, p. $87-96$

\footnotetext{
${ }^{1}$ academie-francaise.fr/linstitution/les-missions

2 culture.gouv.fr/culture/dglf/terminologie/termino_enrichissement.htm

${ }^{3}$ Pour les détails, consulter le site de la Délégation générale à la langue française : dglf.culture.gouv.fr/lois/archives/histoire1.htm

${ }^{4}$ legifrance.gouv.fr/affichTexte.do?cidTexte $=$ LEGITEXT000005621310\&dateTexte $=20100312$

${ }^{5}$ Nommé à sa création « Office de la langue française », l'organisme porte depuis 2002 le nom d' « Office québécois de la langue française ».

${ }^{6}$ oqlf.gouv.qc.ca/office/mission.html

${ }_{8}^{7}$ Petit Robert 2014, version électronique.

${ }^{8}$ Ibid.

${ }^{9}$ Ibid.

${ }^{10}$ Par exemple pin's, rugbyman, speakerine et surbooking, dans le Petit Robert 2014.

${ }^{11}$ www.lepetitrobert.fr/le-petit-robert/comment-les-mots-entrent-ils-dans-le-dictionnaire

${ }^{12}$ REY, Alain et REY-DEBOVE, Josette, "Préface du Nouveau Petit Robert (1993) », reproduite dans Le Petit Robert de la langue française 2014, édition numérique, Dictionnaires Le Robert.

${ }^{13}$ Journal officiel du 20 juin 2003.

${ }^{14}$ Le Nouveau Petit Robert de la langue française 2007.

${ }^{15}$ REY, Alain, «Préface de la $2^{\mathrm{e}}$ édition (1984-2001) », reproduite dans Le Grand Robert de la langue française 2013, édition numérique, Dictionnaires Le Robert.

${ }^{16}$ Journal officiel du 5 avril 2006.

${ }^{17}$ Ibid.

${ }^{18}$ Le Nouveau Petit Robert, dictionnaire alphabétique et analogique de la langue française, 1996.

${ }^{19}$ Le Nouveau Petit Robert, dictionnaire alphabétique et analogique de la langue française, 2004.

${ }^{20}$ Journal officiel du 25 mars 2006.

${ }^{21}$ Ainsi que nous l'avons indiqué plus haut, l'Office de la langue française est devenu en 2002 l'Office québécois de la langue française. Cette mise à jour n'a pas été faire dans Le Grand Robert 2013.

${ }^{22}$ Usito est un dictionnaire général de langue française fait au Québec par le groupe de recherche Franqus de

l'Université de Sherbrooke (dont est membre l'auteure de cet article). Il est disponible en ligne : usito.com/dictio/accueil.zul et a été lancé en mars 2013.

${ }^{23}$ Cette banque est connue en Europe sous le nom d'Europresse.com.

${ }^{24}$ Dans ce dernier cas, il faudrait bien sûr distinguer alors si le mot est réellement neutralisé, ou s'il est utilisé en italique, entre guillemets, suivi d'une traduction, etc.
} 